\title{
A topological method for the classification of entanglements in crystal networks
}

\author{
Eugeny V. Alexandrov, Vladislav A. Blatov and Davide M. Proserpio
}

Acta Cryst. (2012). A68, 484-493

Copyright (C) International Union of Crystallography

Author(s) of this paper may load this reprint on their own web site or institutional repository provided that this cover page is retained. Republication of this article or its storage in electronic databases other than as specified above is not permitted without prior permission in writing from the IUCr.

For further information see http://journals.iucr.org/services/authorrights.html

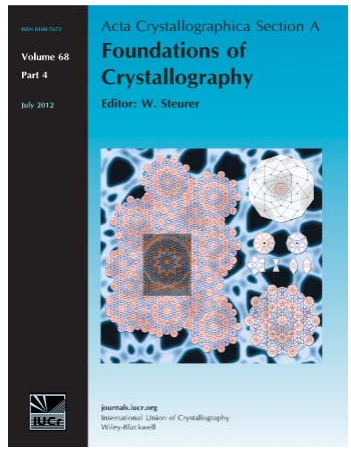

Acta Crystallographica Section A: Foundations of Crystallography covers theoretical and fundamental aspects of the structure of matter. The journal is the prime forum for research in diffraction physics and the theory of crystallographic structure determination by diffraction methods using X-rays, neutrons and electrons. The structures include periodic and aperiodic crystals, and non-periodic disordered materials, and the corresponding Bragg, satellite and diffuse scattering, thermal motion and symmetry aspects. Spatial resolutions range from the subatomic domain in charge-density studies to nanodimensional imperfections such as dislocations and twin walls. The chemistry encompasses metals, alloys, and inorganic, organic and biological materials. Structure prediction and properties such as the theory of phase transformations are also covered.

\section{Crystallography Journals Online is available from journals.iucr.org}


Acta Crystallographica Section A

Foundations of

Crystallography

ISSN 0108-7673

Received 12 February 2012

Accepted 27 April 2012

(C) 2012 International Union of Crystallography

Printed in Singapore - all rights reserved

\section{A topological method for the classification of entanglements in crystal networks ${ }^{1}$}

\author{
Eugeny V. Alexandrov, ${ }^{a}$ Vladislav A. Blatov ${ }^{a}$ and Davide M. Proserpio ${ }^{b_{*}}$ \\ ${ }^{\mathbf{a}}$ Samara State University, Ac. Pavlov St. 1, Samara, 443011, Russian Federation, and ${ }^{\mathbf{b}}$ Dipartimento \\ di Chimica Strutturale e Stereochimica Inorganica (DCSSI), Università degli Studi di Milano, Via G. \\ Venezian 21, 20133, Milano, Italy. Correspondence e-mail: blatov@samsu.ru, \\ davide.proserpio@unimi.it
}

\begin{abstract}
A rigorous method is proposed to describe and classify the topology of entanglements between periodic networks if the links are of the Hopf type. The catenation pattern is unambiguously identified by a net of barycentres of catenating rings with edges corresponding to the Hopf links; this net is called the Hopf ring net. The Hopf ring net approach is compared with other methods of characterizing entanglements; a number of applications of this approach to various kinds of entanglement (interpenetration, polycatenation and self-catenation) both in modelled network arrays and in coordination networks are considered.
\end{abstract}

\section{Introduction}

Entanglement in crystal structures is a fascinating phenomenon that has been intensively investigated since the 1990s when Robson and co-workers (Hoskins \& Robson, 1990; Batten \& Robson, 1998) drew attention to this part of crystal chemistry. To the best of our knowledge, the first review of interpenetrating networks both in inorganic and organic crystals (such as cuprite $\mathrm{Cu}_{2} \mathrm{O}$ or $\beta$-quinol) was done by Wells (1954); however, a long time passed before the investigations of chemically unbonded but spatially non-separable motifs became important to chemists (Batten \& Robson, 1998). Now the number of examples of merely three-dimensional interpenetration is almost 1000 (Alexandrov et al., 2011), while other types of entanglements have not been comprehensively catalogued.

The topological properties of entangled motifs were in focus from the very beginning. It was clear that one of the important properties was the periodicity of interlocking networks as well as of the resulting whole entangled array. Wells (1954) described all kinds of entanglements between three-periodic networks known at that time. Batten \& Robson (1998) introduced the terms inclined and parallel interpenetration for different methods of interlocking two-periodic networks. Batten $(2001,2010)$ proposed to describe entanglements with the formula $n \mathrm{D} / m \mathrm{D} \rightarrow k \mathrm{D}$ (now the form $n \mathrm{D}+m \mathrm{D} \rightarrow k \mathrm{D}$ is more useful) where $m, n$ are periodicities of the entangled networks, and $k$ is the periodicity of the whole array. Carlucci et al. (2003) and Proserpio (2010) distinguished three types of entanglements: interpenetration, when $m, n$ coincide with $k$; polycatenation, when $m, n<k$; and self-catenation (other equivalent terms are self-penetration or polyknotting, $c f$.

\footnotetext{
${ }^{1}$ A preliminary account of this work was presented at the workshop 'Topological dynamics in physics and biology' held in Pisa, 12-13 July 2011.
}

Jensen et al., 2000; Ke et al., 2011). A self-catenated network exhibits the peculiar feature of containing rings through which pass other components of the same network (see below for details). Carlucci et al. (2003) also proposed two kinds of additional topological parameters: degree of catenation (Doc, that is the number of networks entangled to a particular one) and index of separation (Is, that is the number of networks that should be removed to disjoint the array into two unconnected parts) that allowed them to classify the entanglements more thoroughly.

The first attempt to algorithmize the classification of entanglements was undertaken by Blatov et al. (2004), who developed a rigorous computer procedure to characterize three-dimensional interpenetration by the degree of interpenetration (the number of entangled networks) and a number of space-symmetry parameters. This procedure was used to catalogue 301 interpenetrated metal-organic frameworks from the Cambridge Structural Database (Allen, 2002). This approach has also been applied for inorganic and hydrogenbonded networks and is routinely used by experimentalists to analyse three-periodic interpenetration (Baburin et al., 2005, $2008 a, b)$. However, the classification criteria of Blatov et al. (2004) are mainly geometrical and do not concern the details of interlocking the entangled motifs. They introduce classes of interpenetration in terms of crystallographic symmetry relationships which do not recognize topologically different interpenetrating network arrays that have the same sets of the space-symmetry parameters (i.e. for a given net, in the same class of interpenetration there may be different entanglements). A true topological description of interpenetration should be free of crystallographic symmetry relationships which may be affected by non-topological factors such as molecular geometries, the presence and placement of guest species etc. 
A more detailed classification of all homogeneous twoperiodic and three-periodic interpenetrating sphere packings in cubic, hexagonal and tetragonal crystal systems was developed by Fischer \& Koch (1976) and Koch et al. (2006). They proposed the term interpenetration pattern to distinguish different methods of interlocking networks irrespective of the network parts (rings) not participating in the entanglement and of the size of the entangled parts. All interpenetration patterns of the homogeneous sphere packings were tabulated, but practical application of the approach was hindered because no clear parameters of the patterns were proposed that would allow one to detect them in crystal structures.

In this paper, we propose a rigorous method to characterize entanglements. The method is algorithmized and implemented as a computer routine that makes it useful for distinguishing and classifying entanglements of any complexity in periodic network arrays.

\section{The method}

Let us restrict our consideration to the entanglements caused only by Hopf links and/or multiple crossing links [as observed in interpenetrated quartz networks (Delgado-Friedrichs et al., 2003)] between the network cycles. As both Hopf and multiple crossing links are pairwise (they occur between two rings, Fig. 1), below we refer always to Hopf links meaning multiple crossing links too, if not otherwise specified. Thus we avoid, for the present, the multi-ring Brunnian interlockings including the Borromean entanglement (Fig. 1), which rarely occur in crystal structures (Carlucci et al., 2003). We will use the term catenation for the Hopf link as this method of interlocking characterizes the class of organic molecules catenanes. Further, we consider the Hopf links only between strong rings, i.e. cycles that cannot be represented as sums of smaller cycles (Delgado-Friedrichs \& O'Keeffe, 2005). Unlike cycles, the number of which is in general infinite, the set of symmetry non-equivalent strong rings is always finite (Goetzke, 1993). Moreover, strong rings characterize all smallest windows in the network (Blatov et al., 2007) and contain a cycle basis (any cycle in the network is either a strong ring or a sum of strong rings), so they provide sufficient information required to describe the overall catenation. Below we will call them rings for short.

If we then represent each ring by its barycentre and connect the barycentres of catenating rings we obtain what we call the Hopf ring net $(\mathrm{HRN})$, i.e. the net whose nodes and edges correspond to rings and Hopf links between them. The following properties of the HRN are worth mentioning: (i) the degree (coordination number) of a node is equal to the number of rings catenating a particular ring; (ii) some nodes can have the same coordinates if the barycentres of the corresponding rings coincide (i.e. the nodes collide); (iii) the star of a node

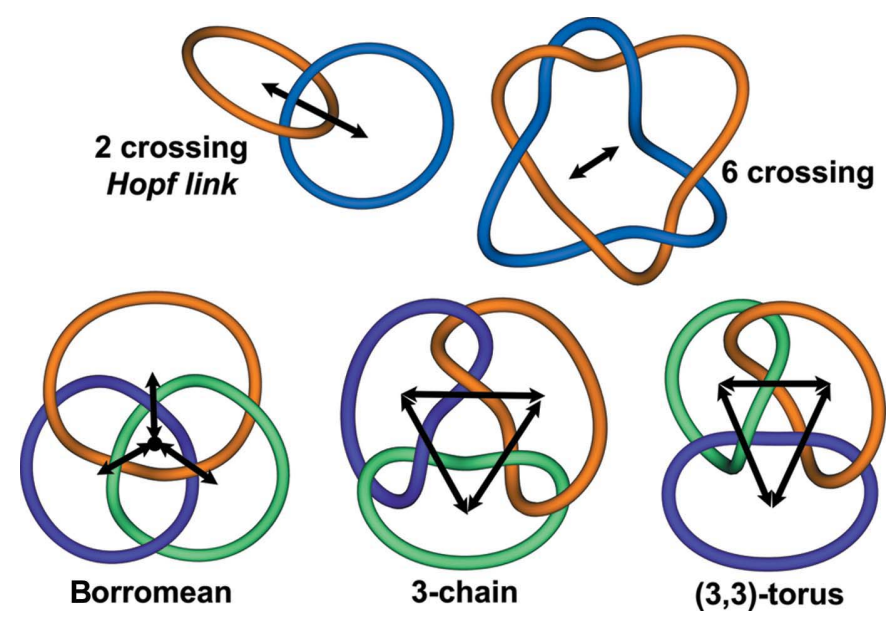

Figure 1

Hopf, multiple crossing and the three simplest three-component links. The corresponding edges of the ring nets that connect the ring-net nodes are shown by arrows. For the Borromean link, the ring-net fragment contains an additional node in the centre of the link. The program Knotplot (R. G. Scharein; http://www.knotplot.com/) was used to draw the link pictures.

defines the bouquet of catenating rings (by bouquet we mean the union of a particular ring and all rings that catenate this selected one), which can be considered as the smallest collection of catenated rings that characterize the entanglement (Fig. 2).

The concept of the Hopf ring net extends the notion of complete ring net (CRN) introduced by Baburin \& Blatov (2007) for single nets. In general, the CRN is derived from a particular network in the following way: the nodes of the CRN correspond to the barycentres of all rings in the initial network, while the edges of the CRN connect the rings that are in contact in the initial network. The method of CRN construction depends on the definition of such contacts (i.e. edges). Baburin \& Blatov (2007) treated the rings as connected if they had common edges with the initial network reference ring. In this work we extend the notion of CRN to include the presence of Hopf links either from entanglement of different networks and/or from self-catenation, adding also the edges for the catenation of the Hopf links but assigning them to a different type.

Therefore we can distinguish edges in the CRN according to the type assigned, which corresponds to different methods of

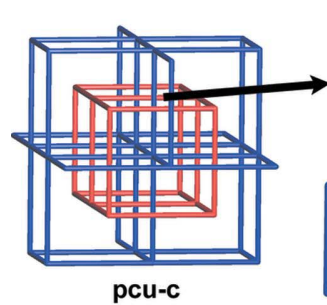

Figure 2

Two interpenetrating primitive cubic (pcu) networks (array pcu-c) shown in red and blue as well as the corresponding $\mathrm{HRN}$ of nbo $(\mathrm{NbO})$ type highlighted in green. The bouquet of catenating rings and the corresponding HRN star (green balls) are shown in the second picture from the left. 
linking rings. In this paper, we distinguish two kinds of edges in the CRN: one corresponds to Hopf links and the other conforms to any connection of rings within the same network through the network vertices and edges (such connection may be provided by one common edge, or just a common vertex, or even an edge chain of any length). A special case arises for a self-catenated network where Hopf links exist between the rings of the same network (see below).

With such different descriptions for the kind of edges, ${ }^{2}$ the CRN of the entanglement can be split into two subnets: a partial ring net, which is derived from the links between the barycentres of the rings belonging to the same network, and the HRN. This representation is useful to explore the periodicity of entanglement between the networks: we can determine the periodicities $n$ and $m$ of two catenating networks ( $n$ and $m$ are equal to the periodicities of the corresponding partial ring nets where Hopf links are ignored) as well as the periodicity $k$ of the CRN (i.e. the periodicity that includes the presence of both kinds of links between rings); the method of entanglement can then be written as $n \mathrm{D}+m \mathrm{D}$ $\rightarrow k \mathrm{D}$. It must be noted that the periodicity $h$ of the HRN is limited by $k(h \leq k)$, but can be higher than $n$ or $m$ (see examples of polycatenation below).

The HRN directly characterizes the catenation pattern, i.e. the method of catenation of the rings, if the kind of network and the degree of interpenetration are fixed. For example, if a set of structures containing two interpenetrating diamondoid networks is under consideration, it is sufficient to compare their HRNs to find the differences in their catenation patterns. However, to match catenation patterns of topologically different interpenetrating arrays (the number of interpenetrating networks must be fixed anyway) their HRNs should be reduced beforehand with the two simplification procedures described below.

First, in general, not all rings are catenated, and hence not all of them are represented in the HRN. If a catenated ring $A$ is a sum of another catenated ring $B$ and any number of noncatenated rings, then $A$ and $B$ are equally catenated and can be replaced with a single ring in the HRN. This operation is equivalent to fusing the HRN nodes corresponding to $A$ and $B$. Indeed, such equally catenated rings do not carry any new information about the entanglement and can be treated as the same ring in the catenation pattern. In the HRN, such rings can be detected as collisions (Delgado-Friedrichs \& O'Keeffe, 2005); the corresponding nodes have the same set of neighbours. For instance, three distinct types of 14-rings in dia-f (that is a decorated version of the diamond network) are almost coincident (they share 12 vertices of the 14; see Fig. 3, left). As a result they have three distinct but almost coincident barycentres (the three green spheres) giving rise to three superimposed stars. Because each 14-ring is catenated by the same set of 18 (six triplets) other 14-rings and share the same stars in the HRN, the corresponding triplets of the HRN nodes

\footnotetext{
${ }^{2}$ In practice, TOPOS (Blatov, 2006; http://www.topos.samsu.ru) assigns to 'Valence' the edges between the ring barycentres and to 'Hydrogen bond' the Hopf link edges.
}

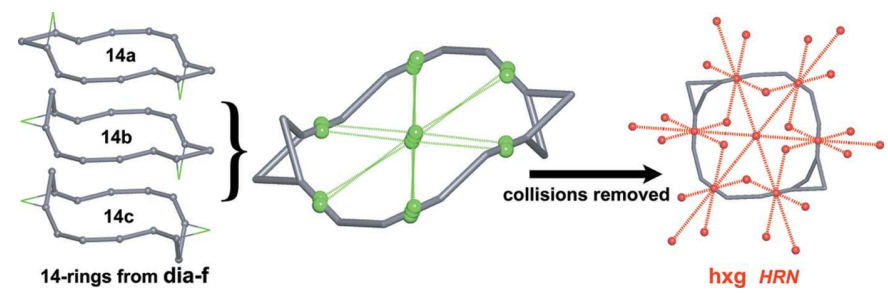

Figure 3

Three equally catenated 14-rings in an interpenetrating array of two decorated diamondoid (dia-f) networks and the corresponding fragment of the simplified HRN. All the 14-rings are related via non-catenated 4-rings: $14 a$-ring $=14 b$-ring +4 -ring $=14 c$-ring +4 -ring +4 -ring $($ see text for details).

collide (Fig. 3, middle). After removing collisions each triplet collapses into one node 6 -coordinated $(18 / 3=6)$ resulting in an HRN of the hxg topology (Fig. 3, right).

Second, not all remaining rings in each interpenetrating network are independent; some of them are still sums of several catenated rings. Obviously, such dependent rings also do not carry any new information on the catenation. So one should consider only rings from the ring basis, i.e. the minimal set of rings that generate other rings by summation. To the best of our knowledge, there is no general algorithm to determine the ring basis for a periodic network, but a partial and useful solution of this problem may be found within the natural tiling approach. According to Blatov et al. (2007) the natural tiling is the unique method to represent a network as a set of cages (natural tiles) that are confined by strong rings of a special kind called essential rings. An important property of an essential ring is that any strong ring is either an essential ring or a sum of essential rings, i.e. the set of essential rings can be treated as a ring basis. The problem is that there are networks that do not admit the natural tiling, but they are mostly unimportant for crystal chemistry because of their rare occurrence. Indeed, all abundant three-dimensional underlying networks in inorganic, organic or metal-organic compounds (see Alexandrov et al., 2011 and references therein) admit natural tilings [for tilings of networks see the Reticular Chemistry Structure Resource (RCSR), http:// rcsr.anu.edu.au/; O'Keeffe et al., 2008].

Notwithstanding the fact that the method is applicable to any set of given rings, so it could also be used to analyse entanglements for nets that do not admit natural tilings. All the networks considered in this paper, except specially discussed examples of self-catenation, admit the natural tiling, so we will use this approach to determine the ring basis. Resuming, before comparing two HRNs (i.e. two catenation patterns), they should be pruned of collisions and the nodes corresponding to inessential rings. The HRN simplified in this way unambiguously determines the topology of the catenation pattern if the ambient isotopy is not taken into account. Recall that ambient isotopic networks can be superposed in the space without breaking edges or rings (Hyde \& Delgado-Friedrichs, 2011). For example, 3-chain and (3,3)-torus links (Fig. 1) are not ambient isotopic but give rise to equivalent HRN fragments. One can state that two entangled arrays of networks 
have the same catenation patterns if and only if they have isomorphic simplified HRNs. The isomorphism of HRNs can be checked with the methods developed for periodic nets (Blatov, 2007); if the HRN is a finite graph, the corresponding methods from graph theory should be used. The TOPOS TTD collection (Blatov \& Proserpio, 2009) can be used to assign the name to the net topology; this collection currently contains more than 72000 net topologies. Thus the method is purely topological; the catenation patterns can be classified irrespective of the space-group symmetry and geometrical embedding of the entangled networks. It is important that the HRN topology is independent of the size of catenating rings as well as of the number and size of non-catenating rings in the entangled networks. For example, the extension of edges or the decoration of nodes (without the addition of new Hopf links) in the networks does not influence the resulting HRN.

Fig. 4 illustrates the similarity of interpenetration in nine different twofold network arrays (eight of them are interpenetrating sphere packings, see below). Here we consider some possible interpenetrations; the detailed analysis of all known catenation patterns for a given set of interpenetrating networks is in progress and will be discussed elsewhere. The local similarity is obvious from their bouquets, which lead to the same star of five ring barycentres ( $c f$. Fig. 2). Only one interpenetrating array which consists of two peu networks is fully catenated, i.e. has all rings catenated; in all other cases there are either inessential rings (cab, nbo) or some essential rings are not catenated (afw, cab, nbo-a, pcu-g, pcu-h, uku, unp). Only in two cases (uku, unp) are there links that lead to collisions to be removed (Table 1). Nonetheless, the simplification procedure, i.e. removing both collisions and the nodes corresponding to inessential rings, gives rise to the same simplified HRN nbo for all the arrays, which proves that there is the same catenation pattern in all cases.

We have algorithmized the method and implemented it in the program package TOPOS (Blatov, 2006; http://www. topos.samsu.ru). TOPOS provides the analysis of catenation patterns according to the following general algorithm:

(i) searching for all cycles in the network array and selecting strong rings [see Blatov (2006) for details];
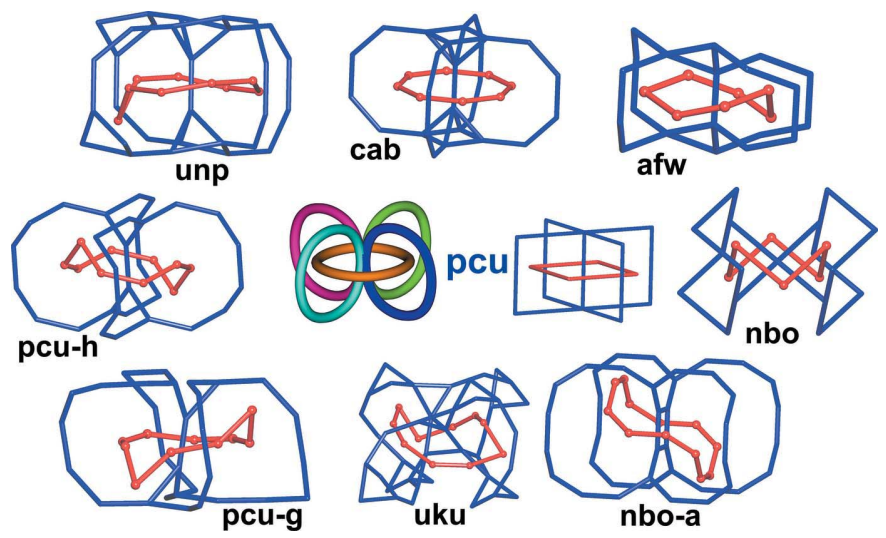

Figure 4

The bouquets of catenating rings in nine different network arrays that lead to the same star of simplified HRN of the nbo topology ( $c f$. Fig. 2).
Table 1

Strong and essential rings in twofold interpenetrating arrays of some networks.

\begin{tabular}{llll}
\hline Network & $\begin{array}{l}\text { Sizes of } \\
\text { strong rings }\end{array}$ & $\begin{array}{l}\text { Sizes of } \\
\text { essential rings }\end{array}$ & $\begin{array}{l}\text { Sizes of catenated } \\
\text { essential rings }\end{array}$ \\
\hline pcu $\dagger$ & 4 & 4 & 4 \\
nbo & 6,8 & 6 & 6 \\
afw (pcu-n) & 3,7 & 3,7 & 7 \\
cab (pcu-a) & $3,4,8$ & 3,8 & 8 \\
unp (pcu-p) & $3,4,9$ & $3,4,9$ & 9 \\
pcu-g & 6,10 & 6,10 & 10 \\
pcu-h $\dagger$ & 6,10 & 6,10 & 10 \\
uku & $3,4,6,10$ & $3,4,6,10$ & 10 \\
nbo-a $\dagger$ & 4,12 & 4,12 & 12 \\
\hline
\end{tabular}

$\dagger$ Array is observed in real structures.

(ii) searching for all entanglements and selecting Hopf links [see Blatov (2006) for details];

(iii) distinguishing Hopf links between different networks (catenation) and within the same network (self-catenation);

(iv) constructing an HRN;

(v) removing collisions from the HRN (merging the nodes);

(vi) determining essential rings in accordance with the algorithm by Blatov et al. (2007) and removing all HRN nodes that correspond to inessential rings;

(vii) determining the HRN topology according to Blatov (2007).

\section{Examples}

Below we consider different kinds of network arrays and methods of entanglement to demonstrate the applicability of the HRN approach. To designate nets, we use bold three-letter RCSR symbols (e.g. dia for the diamondoid network) and the Fischer $k / m / f n$ nomenclature for sphere packings (Koch et al., 2006).

\subsection{Networks admitting naturally self-dual tilings}

Most naturally, twofold interpenetration is realized in networks that admit a self-dual tiling: the networks are isomorphic to their duals (Delgado-Friedrichs et al., 2007). By definition, in the naturally dual network, the nodes, edges, essential rings and natural tiles are in one-to-one relation to natural tiles, essential rings, edges and nodes of the network under consideration. The simplified HRNs (catenation patterns) for twofold arrays of 23 interpenetrating naturally self-dual networks which are stored in the RCSR database are given in Table 2. The crystallographic data on the interpenetrated arrays for cds, dia, hms, pcu, pyr, sda, srs and tfa were taken from the RCSR database (reported with the suffix -c). For the other self-dual networks the interpenetrated arrays have not yet been found in crystal structures (with the exception of sxd); no data on their embeddings are available so far. In this case we have generated an embedding using the TOPOS procedure for constructing dual nets (Blatov et al., 2007). For one twofold array of naturally selfdual networks $[(4,4)$-coor bbr] we could not find any faithful 
Table 2

Simplified Hopf ring nets for twofold arrays of interpenetrating naturally self-dual networks.

\begin{tabular}{|c|c|c|c|}
\hline Network & Hopf ring net $\dagger$ & Network & Hopf ring net $\dagger$ \\
\hline$(5,7)$-coor cbs $\ddagger$ & $\begin{array}{l}(3,4,4,4,4,5) \text {-coor } \\
395204472 \\
410264680 \\
4123259100 \\
414285086 \\
4143361102 \\
5 \quad 153566 \quad 100\end{array}$ & $(4,5)$-coor rtw $\ddagger$ & $\begin{array}{l}(4,5,6) \text {-coor } \\
4143678134 \\
5194995146 \\
6204994149\end{array}$ \\
\hline 4-coor cds $\S$ & $\begin{array}{l}(6,8) \text {-coor } \\
62870126198 \\
83072128200\end{array}$ & 6-coor qtz-x & 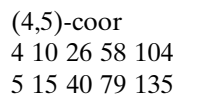 \\
\hline 4-coor dia§ & 6-coor hxg & 6-coor sda & 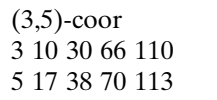 \\
\hline$(3,3)$-coor ete $\ddagger$ & 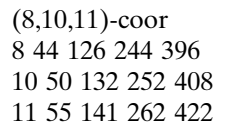 & 6-coor smt $\ddagger$ & $\begin{array}{l}(3,4,7) \text {-coor } \\
3 \begin{array}{lllll}31 & 35 & 74 & 126 \\
4 & 14 & 40 & 84 & 139 \\
7 & 23 & 49 & 84 & 135\end{array}\end{array}$ \\
\hline$(4,6)$-coor fsf $\ddagger$ & 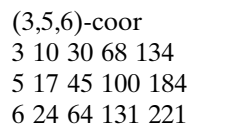 & 3-coor srs $\S$ & $\begin{array}{l}10 \text {-coor } \\
1050130244394\end{array}$ \\
\hline$(4,12)$-coor ftw $\ddagger$ & 4-coor rhr & 7-coor swl $\$$ & $\begin{array}{l}(3,4,4,4) \text {-coor } \\
37173561 \\
411254878 \\
412264676 \\
412285082\end{array}$ \\
\hline$(3,5)$-coor hms $\S$ & $\begin{array}{l}(6,6) \text {-coor } \\
6225496150 \\
6245498150\end{array}$ & 6 -coor sxd $\ddagger \S$ & 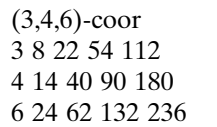 \\
\hline 6-coor mab $\ddagger$ & $\begin{array}{l}(4,6) \text {-coor } \\
4164072112 \\
6184072114\end{array}$ & $(3,4)$-coor $\mathbf{t f a} \S$ & $\begin{array}{l}(8,8) \text {-coor } \\
83692172 \\
836 \\
83693173278\end{array}$ \\
\hline$(3,3,3,4)$-coor mco $\ddagger$ & $\begin{array}{l}(8,8,8) \text {-coor } \\
83694185305 \\
83697185300 \\
83698196308\end{array}$ & $(3,12)$-coor ttv $\neq$ & $\begin{array}{l}(4,5) \text {-coor } \\
412285896 \\
5153671116\end{array}$ \\
\hline 6-coor pcu§ & 4-coor nbo & 4-coor $\mathbf{u n j} \ddagger$ & 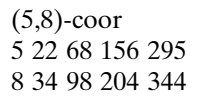 \\
\hline$(4,6)$-coor pte & 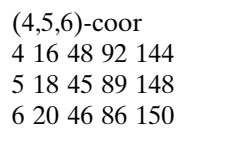 & 7-coor vck & 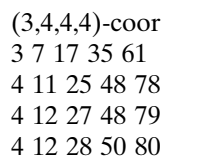 \\
\hline$(3,6)$-coor pyr $\S$ & $\begin{array}{l}\text { 6-coor } \\
62258113190\end{array}$ & & \\
\hline
\end{tabular}

$\dagger$ The first five terms of coordination sequences are given for each independent node if the net topology is not deposited in the TOPOS TTD collection. The abbreviation $\left(n_{1}\right.$, $n_{2}, \ldots$ )-coor means that the net contains topologically non-equivalent nodes with $n_{1}, n_{2}$, ... coordination numbers. \$ No crystallographic data on interpenetrating -c array are available in the RCSR or elsewhere. \& Examples of twofold interpenetrating arrays of this topology were found in crystals (Alexandrov et al., 2011).

embedding (without crossing edges, $c f$. Delgado-Friedrichs et al., 2005).

The simplification procedures are required for the HRNs of the cds, ftw, mab, pte, rtw, qtz-x, smt, swl and vck twofold arrays where not all strong rings are essential. One can notice that for most of the HRNs which describe catenation patterns occurring in crystal structures (cds-c, dia-c, hms-c, pcu-c, pyr-c, srs-c, sxd-c and tfa-c) the topologies are rather simple: no more than three different nodes exist, i.e. the rings in the interpenetrated array are catenated quite uniformly. In the hms, mco, tfa and vck arrays, different rings are catenated in a
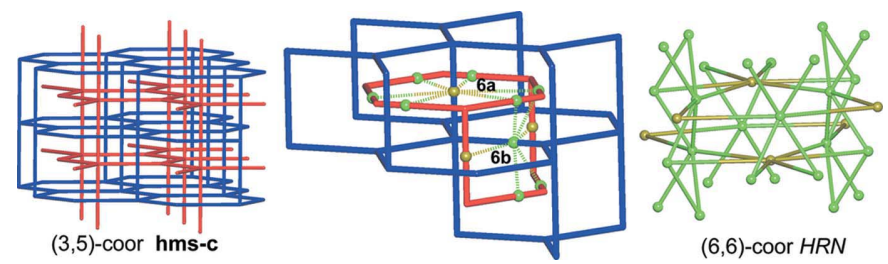

Figure 5

(Left) Twofold (3,5)-coordinated hms array (hms-c), (middle) the bouquet of the catenating rings of the two independent 6-rings $(6 a, 6 b$ in red) with the HRN stars (yellow and green) and (right) a fragment of the corresponding $(6,6)$-coordinated binodal HRN.

very close but different fashion: it follows that the coordination sequences of the corresponding nodes in the HRN are very close. Since the coordination sequences show the numbers of nodes in subsequent coordination shells of a particular node, their similarity means that the catenation patterns are very close in the vicinity of these rings. For example, in the hms-c array each of the two non-equivalent 6-rings is catenated by six other rings (Fig. 5, middle); they correspond to the first coordination shell of the $(6,6)$-coordinated HRN (Table 2). In turn, these six rings catenate 22 or 24 other rings that form second coordination shells around the two non-equivalent 6-rings etc:; the numbers of rings in the subsequent shells of this 'chain mail' do not differ by more than two (Table 2). Obviously, such subtle differences can hardly be revealed by a visual analysis.

One feature of the interpenetrating arrays under consideration follows from the property of naturally self-dual networks: every essential ring of one network is crossed by one and only one edge of the other network, i.e. a twofold array of self-dual networks is fully catenated (all essential rings are catenated). As a result, every essential $n$-ring is catenated by strictly $n$ essential rings of the other network, i.e. the coordination numbers of nodes in the simplified HRN are equal to the sizes of the corresponding rings. For example, a diamondoid (dia) network contains only one kind of essential 6-rings and the corresponding HRN for dia-c is 6-coordinated hxg; the essential rings in an srs $\left(\mathrm{SrSi}_{2}\right)$ network are 10membered and the srs-c array has a 10-coordinated HRN (in every bouquet the $n$-ring is catenated by $n$ other rings, Fig. 6).

\subsection{Interpenetrating sphere packings and coordination networks}

A good test to verify the proposed approach is to compare the catenation patterns in terms of simplified HRNs with the interpenetration patterns of sphere packings by Koch et al. (2006). In Table 3, all interpenetration patterns in 149 threeperiodic sphere packings are matched to 18 simplified HRNs (more detailed information is given in the supplementary material $^{\mathbf{3}}$ ). Recall that the interpenetration pattern symbol $a-$ $b$ indicates the crystal system of the array $(a=c, h, r, t$ or $o$ for

\footnotetext{
${ }^{\mathbf{3}}$ Supplementary material for this paper is available from the IUCr electronic archives (Reference: EO5016). Services for accessing this material are described at the back of the journal.
} 

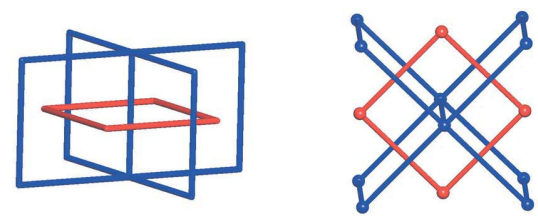

2 fold pcu $=$ pcu-c

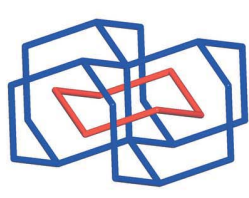

2 fold dia $=$ dia $-c$
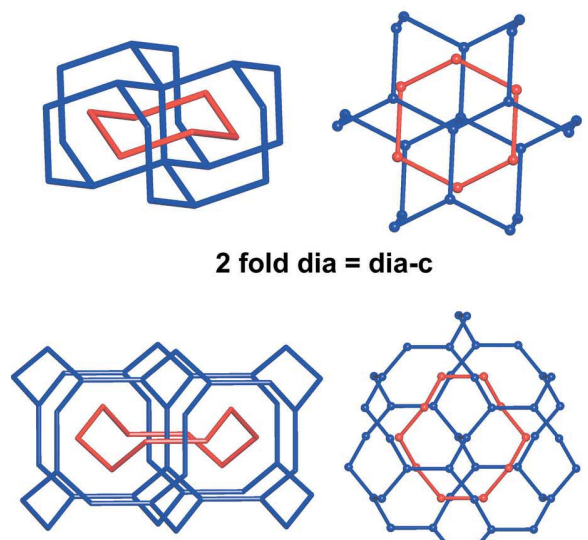

2 fold srs $=$ srs-c

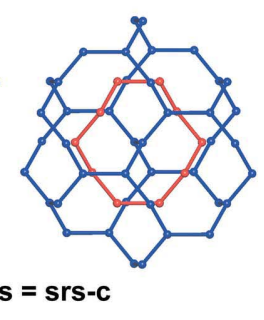

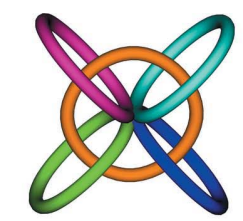
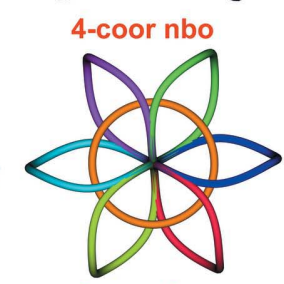

6-coor hxg

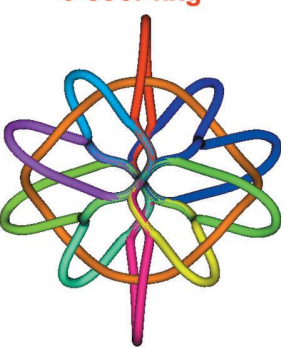

10-coor NEW
Figure 6

Fragments of pcu-c, dia-c and srs-c arrays and the corresponding bouquets.

cubic, hexagonal, rhombohedral, tetragonal or orthorhombic system) and the type of interpenetration ( $b=a-r$ for 18 different types).

Table 3 shows a clear correspondence between interpenetration patterns and simplified HRNs; however, some differences should be noted. Almost each type of interpenetration $(a-r)$ matches a distinct catenation pattern represented by a unique simplified HRN. The only exception is the pair $h-n, h-q$ which describes two quartz-like arrangements that differ by labyrinths of the corresponding minimal surfaces (Koch et al., 2006); the catenation in these arrays has the same HRN (up to ambient isotopy): at this stage we cannot differentiate them (Hyde \& Delgado-Friedrichs, 2011). Note also the type $r-b c$ : according to Koch et al. (2006) it can be related both to $b$ and $c$ interpenetration pattern types since it can be derived both from $c-b$ and $c-c$ patterns by a rhombohedral distortion. Obviously, the distortion of the $c-b$ pattern is followed by a change of catenation; the resulting HRN of the nbo type unambiguously indicates the $c$ type of interpenetration.

The HRN reflects only the topological properties of catenation; therefore the same interpenetration patterns that are realized in different crystal systems conform to the same simplified HRN. This also results in differences in the description of interpenetration patterns in the $t[3 / 4 / t 2]^{2}$ and $t[3 / 4 / t 2]^{2}{ }_{\text {II }}$ sphere packings (corresponding to the twofold dia-g array): Koch et al. (2006) put them into the $t-b$ and $t-a$ types, respectively, owing to a different arrangement of screw axes, while our approach unambiguously relates them to the same catenation pattern of the hxg type that corresponds to the $b$ interpenetration pattern (see the supplementary material). At
Table 3

Interpenetration patterns in three-periodic sphere packings according to Koch et al. (2006) with the degree of interpenetration $(Z)$ and the corresponding simplified Hopf ring nets.

For each pattern we list the number of sphere packings where it appears (No. $\mathrm{SP}$ ) and for how many different network topologies (No. topologies). See the supplementary material for details.

\begin{tabular}{|c|c|c|c|c|}
\hline $\begin{array}{l}\text { Interpenetration } \\
\text { pattern }\end{array}$ & $Z$ & Hopf ring net & No. SP & $\begin{array}{l}\text { No. } \\
\text { topologies }\end{array}$ \\
\hline$c-a, t-a, o-a$ & 2 & $\begin{array}{l}\text { 10-coor } \\
1050130244394\end{array}$ & 34 & 16 \\
\hline$c-b, t-b, o-b$ & 2 & 6-coor hxg & 56 & 16 \\
\hline$c-c, r-b c$ & 2 & 4-coor nbo & 20 & 7 \\
\hline$c-d$ & 2 & $\begin{array}{l}\text { 6-coor } \\
62258113190\end{array}$ & 1 & 1 \\
\hline$c-e$ & 2 & $\begin{array}{l}\text { 19-coor } \\
191264239921813\end{array}$ & 2 & 1 \\
\hline$c-f$ & 2 & $\begin{array}{l}\text { 26-coor } \\
2619663113582303\end{array}$ & 1 & 1 \\
\hline$c-g$ & 4 & $\begin{array}{l}\text { 39-coor } \\
3934811532505 \\
4210\end{array}$ & 1 & 1 \\
\hline$c-h$ & 4 & $\begin{array}{l}\text { 46-coor } \\
4644814082872 \\
4834\end{array}$ & 1 & 1 \\
\hline$c-i$ & 4 & $\begin{array}{l}\text { 34-coor } \\
3432895818823110\end{array}$ & 4 & 1 \\
\hline$c-j$ & 8 & $\begin{array}{l}92 \text {-coor } \\
9210503122\end{array}$ & 1 & 1 \\
\hline$c-k$ & 8 & $\begin{array}{l}80 \text {-coor } \\
8010083014\end{array}$ & 1 & 1 \\
\hline$c-l$ & 3 & $\begin{array}{l}\text { 14-coor } \\
1492298600986\end{array}$ & 2 & 2 \\
\hline$t-m, o-m$ & 3 & $\begin{array}{l}\text { 12-coor } \\
1266192356588\end{array}$ & 12 & 4 \\
\hline$h-n, h-q$ & 2 & $\begin{array}{l}(12,12) \text {-coor } \\
1270224444728 \\
1270228448732\end{array}$ & 6 & 5 \\
\hline$h-o$ & 3 & $\begin{array}{l}(14,16) \text {-coor } \\
14862665761034 \\
16922665681004\end{array}$ & 2 & 2 \\
\hline$t-p$ & 4 & $\begin{array}{l}\text { 18-coor } \\
18108310578954\end{array}$ & 2 & 2 \\
\hline$t-r$ & 5 & $\begin{array}{l}\text { 24-coor } \\
241725149821638\end{array}$ & 1 & 1 \\
\hline $\begin{array}{l}t[3 / 10 / c 1]^{2}{ }_{\text {II }}(t-b) \\
\text { twofold srs }\end{array}$ & 2 & $\begin{array}{l}(10,16) \text {-coor } \\
1056160312508 \\
1680198358566\end{array}$ & 2 & 1 \\
\hline
\end{tabular}

the same time, two sphere packings $t[3 / 10 / c 1]^{2}$ II of $P 4_{3} 22$ and $I 4_{1} 22$ space-group symmetries (corresponding to the twofold srs array) fall into the $t-b$ interpenetration pattern type according to Koch et al. (2006), while they have unique simplified HRNs and hence a special catenation pattern (Table 3).

We have shown so far that HRNs correctly characterize the catenation patterns in the three-periodic interpenetrating arrays. This allows one to apply them to classify the catenation patterns in almost 1000 examples of interpenetrating threedimensional coordination networks (Alexandrov et al., 2011). Detailed analysis of this group lies beyond the scope of this paper, but one can mention that the most abundant catenation patterns (twofold dia, pcu and srs, which are also deposited in the RCSR database as dia-c, pcu-c and srs-c) are already described in Table 2. To explore catenation in coordination 


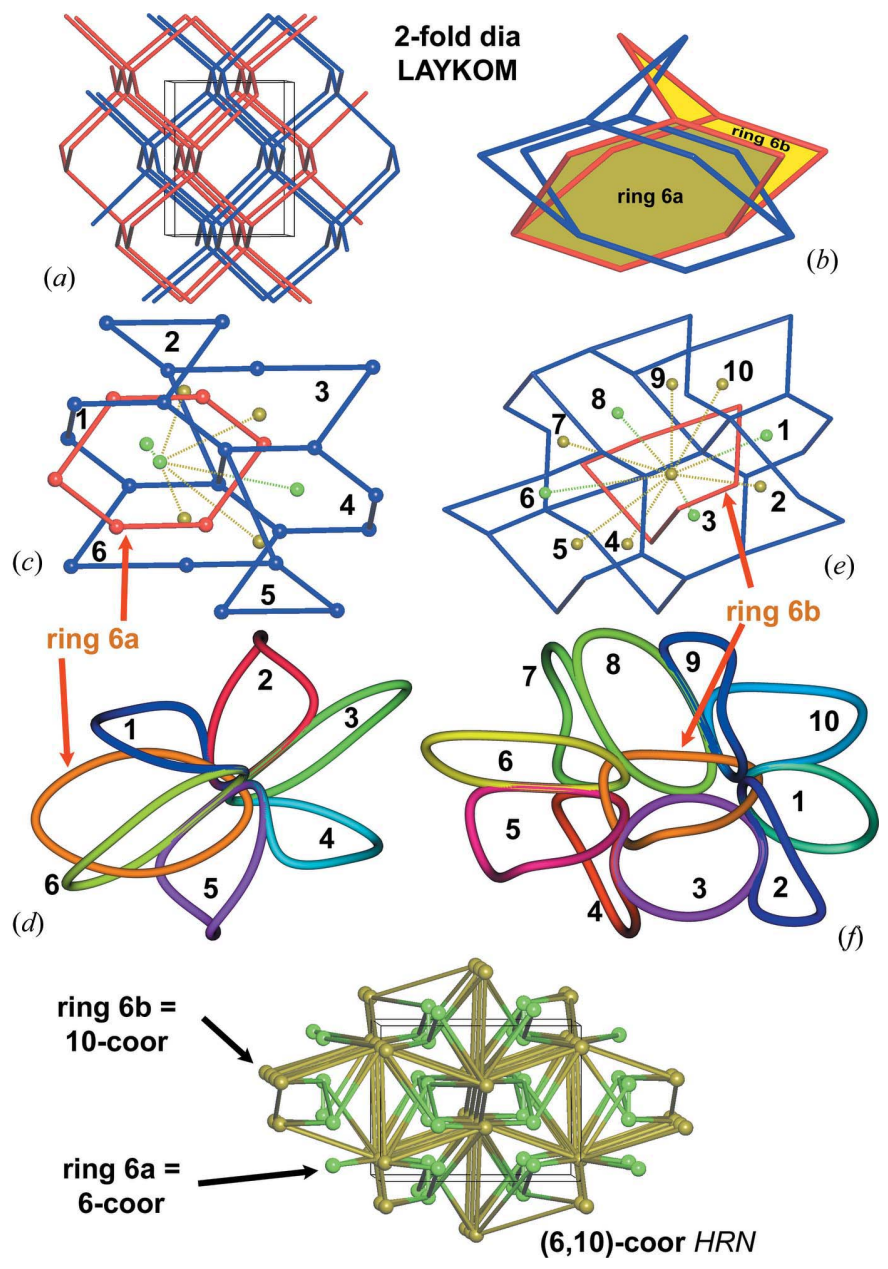

Figure 7

Two different views of the twofold dia array observed in LAYKOM: (a) shows seemingly regular adamantane-like fragments, but another view (b) makes distortion evident (see Fig. 6, dia-c, for comparison); there are two non-equivalent 6-rings $(6 a, 6 b) ;(c),(d)$ and $(e),(f)$ show the corresponding HRN stars and the bouquets that result in the two 6coordinated (green) and 10-coordinated (yellow) HRN nodes. The corresponding 6,10-coordinated HRN is at the bottom.

networks we simplify them into underlying nets that carry the information on the connectivity between structural groups (Alexandrov et al., 2011). Note that the interpenetration in chemical structures is much more diverse compared to modelled systems of spheres; for example, a twofold dia array occurs in hundreds of examples in many different space groups and in two different classes of interpenetration ( $\mathrm{I} a$ and II $a$ ); nonetheless, all of them (but one) belong to the hxg type of catenation (Table 2). A different pattern was found for a unique case that has a binodal 6,10-coordinated $\mathrm{HRN}$ in $\left(\mu_{2^{-}}\right.$ 1,3-di(4-pyridyl)propane)- $\left(\mu_{2}\right.$-5-nitroisophthalato $) \mathrm{Ni}\left(\mathrm{H}_{2} \mathrm{O}\right)$ (LAYKOM; Xiao et al., 2005). ${ }^{4}$ In this case, besides rings catenated by six other rings (which would correspond to the hxg type), there are multiple links between some 6-rings that are ten times catenated (Fig. 7).

\footnotetext{
${ }^{4}$ Hereafter, for each structure, we specify the Cambridge Structural Database refcodes along with the references to the original publications.
}
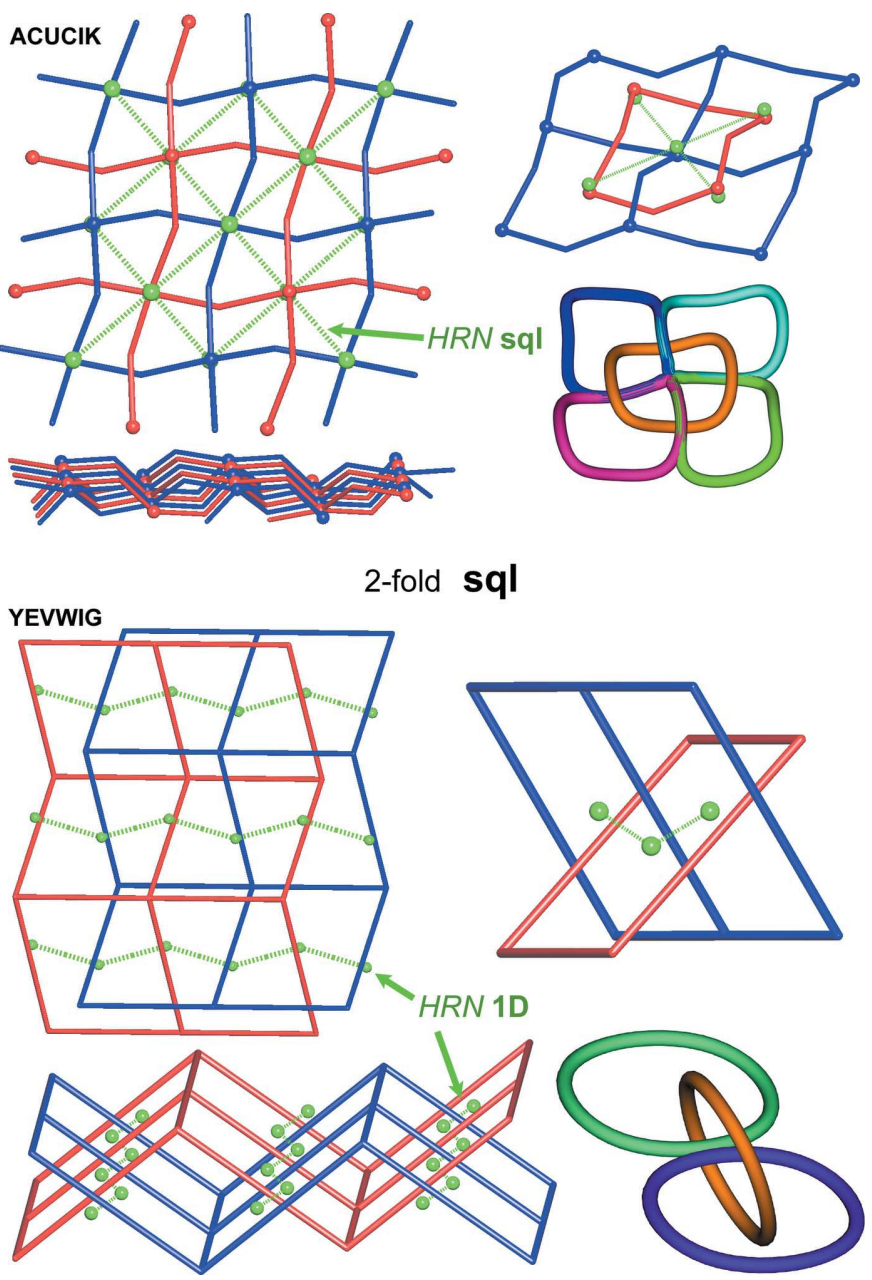

Figure 8

Twofold arrays of square (sql) networks catenated in two different fashions: (top) square plane sql HRN in ACUCIK and (bottom) onedimensional zigzag HRN in YEVWIG. In both cases the bouquets of catenating 4-rings and the corresponding HRN stars are shown.

Similarly, one can consider low-periodic interpenetration. For example, the most typical interpenetration for twoperiodic coordination networks, a twofold array of square (sql) networks, has the HRNs of the same sql topology if every 4-ring of one network is crossed by four 4-rings of the other network as in [( $\mu_{2}-5$-(2-(3-pyridyl)ethenyl)thiophene-2carboxylato) ${ }_{2} \mathrm{Zn}$ ] (ACUCIK; Evans \& Lin, 2001) (Fig. 8, top). This kind of interpenetration reflects the fact that sql is a naturally self-dual two-periodic network. Note that the interpenetration is realized owing to corrugation of the 4-rings and layers caused by additional two-coordinated nodes that correspond to organic ligands. With these nodes the rings become 8-membered, but this extension does not influence the HRN topology as was mentioned above. However, this pattern is not the only one for twofold sql arrays; for example, in [ $\left(\mu_{2^{-}}\right.$ isonicotinato)( $\mu_{2}$-nicotinato)Zn] (YEVWIG; Kang et al., 2007) the HRN consists of an infinite number of zigzag chains because each ring is catenated by only two rings of the other network (Fig. 8, bottom). 


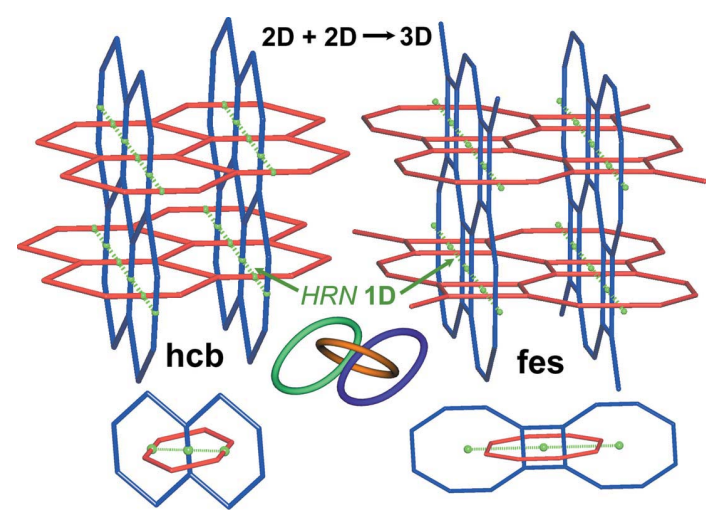

Figure 9

Inclined polycatenation of (left) hcb and (right) fes two-periodic networks and the corresponding one-dimensional linear chain HRNs. The 4-rings in fes do not participate in links and do not contribute to the HRN; their centres coincide with the centres of 8-rings of another fes network.

\subsection{Polycatenation}

As was mentioned above, the polycatenation phenomenon features the arrays where the periodicities $m, n$ of interweaving networks are less than the periodicity $k$ of the whole array. This is the case of interpenetrating two-periodic hcb $\left(6^{3}\right)$ and fes $\left(4.8^{2}\right)$ layers of spheres (Koch et al., 2006) that all have the same chain-like HRNs since each catenated ring (6-ring in hcb and 8-ring in fes) is linked to two similar rings of the other layer (Fig. 9). According to Koch et al. (2006), all inter-

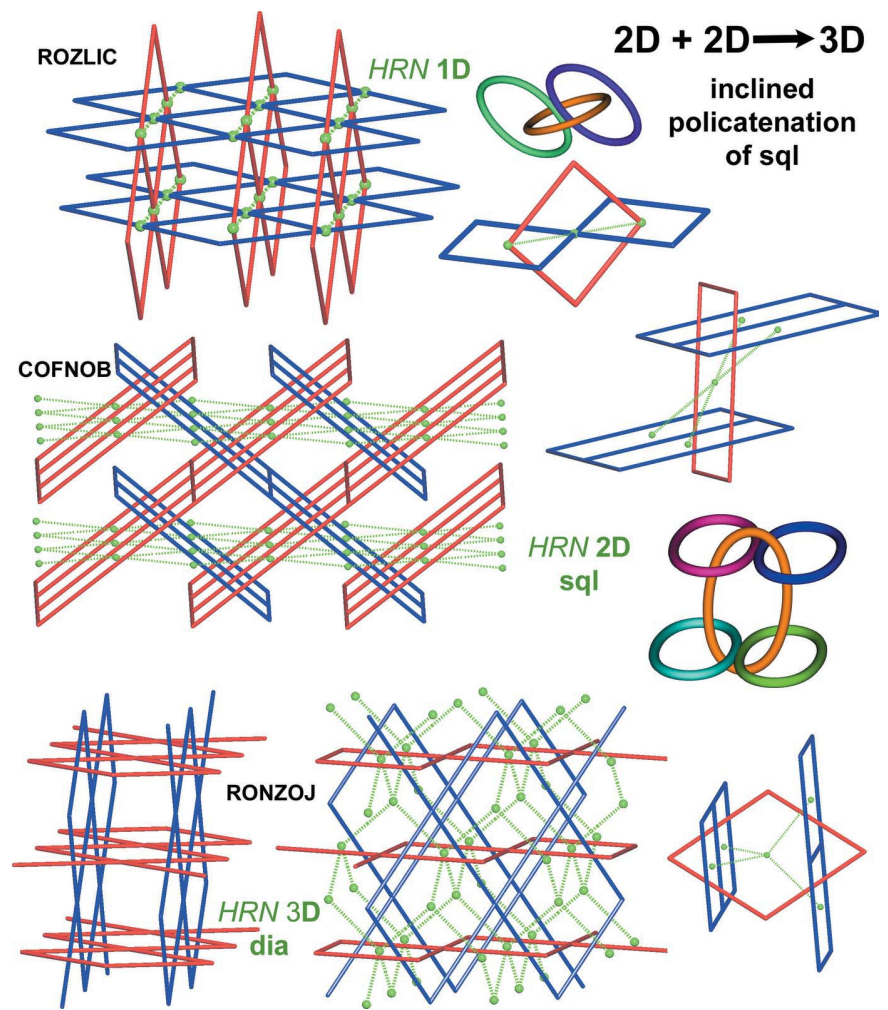

Figure 10

Inclined polycatenation in (top) ROZLIC, ROZLOI; (middle) COFNOB, COFNUH, COFPAP; (bottom) RONZOJ, RONZUP underlying nets and the corresponding HRNs, bouquets and HRN stars. penetrating two-periodic layers of spheres have interpenetration patterns of the same type.

Again, polycatenation of coordination networks is much more diverse. The interpenetrating layers of spheres represent only one, inclined type of polycatenation when the interweaving two-periodic networks are non-parallel. However, even polycatenated arrays of this type can have quite different HRNs. For example, inclined polycatenation of sql layers can adopt both a chain-like $\mathrm{HRN}$ motif as in [ $\left(\mu_{2}-4,4^{\prime}\right.$-bipyridine) $\left.\mathrm{Cu}\left(\mathrm{H}_{2} \mathrm{O}\right)\right]_{2} M \mathrm{~F}_{6}(M=\mathrm{Mo}, \mathrm{Nb})$ (ROZLIC, ROZLOI; Mahenthirarajah et al., 2009) and a layer-like HRN motif of the sql topology as in [( $\mu_{2}$-bis(4-pyridylmethyl)piperazine $)\left(\mu_{2}\right.$-succinato $\left.) M\left(\mathrm{H}_{2} \mathrm{O}\right)_{2}\right](M=\mathrm{Co}, \mathrm{Ni}, \mathrm{Cu})$ (COFNOB, COFNUH, COFPAP; Martin et al., 2008), or even a threeperiodic dia-type HRN motif as in clathrates of [(pyridine $\left.)_{2} \mathrm{Cd}\left\{\mathrm{Ag}(\mathrm{CN})_{2}\right\}_{2}\right] \quad$ (RONZOJ, RONZUP; Soma \& Iwamoto, 1996) (Fig. 10). In the last example, the HRN periodicity is higher than the periodicity of catenating networks.

The parallel type of polycatenation also admits various topologies of the corresponding HRNs. Thus polycatenated sql layers can adopt two-periodic HRNs of the same sql topology as in [( $\mu_{2}-1,3$-bis(imidazol-1-ylmethyl)benzene $)_{2}\left(\mu_{2^{-}}\right.$ terephthalato $)_{2} \mathrm{Zn}_{2}$ ] (GIMGIT; Tian et al., 2007) or of threeperiodic 4-coordinated Ivt topology as in hydrates of $\left[\left(\mu_{2^{-}}\right.\right.$ pyridine-2,3-dicarboxylato)-( $\mu_{2}-1,3$-bis(4-pyridyl)propane $\left.) M\right]$ $(M=\mathrm{Cu}, \mathrm{Zn})$ (BONNEY, BONNOI; Wang et al., 2009) (Fig. 11). For the latter example, as for the twofold sql ACUCIK

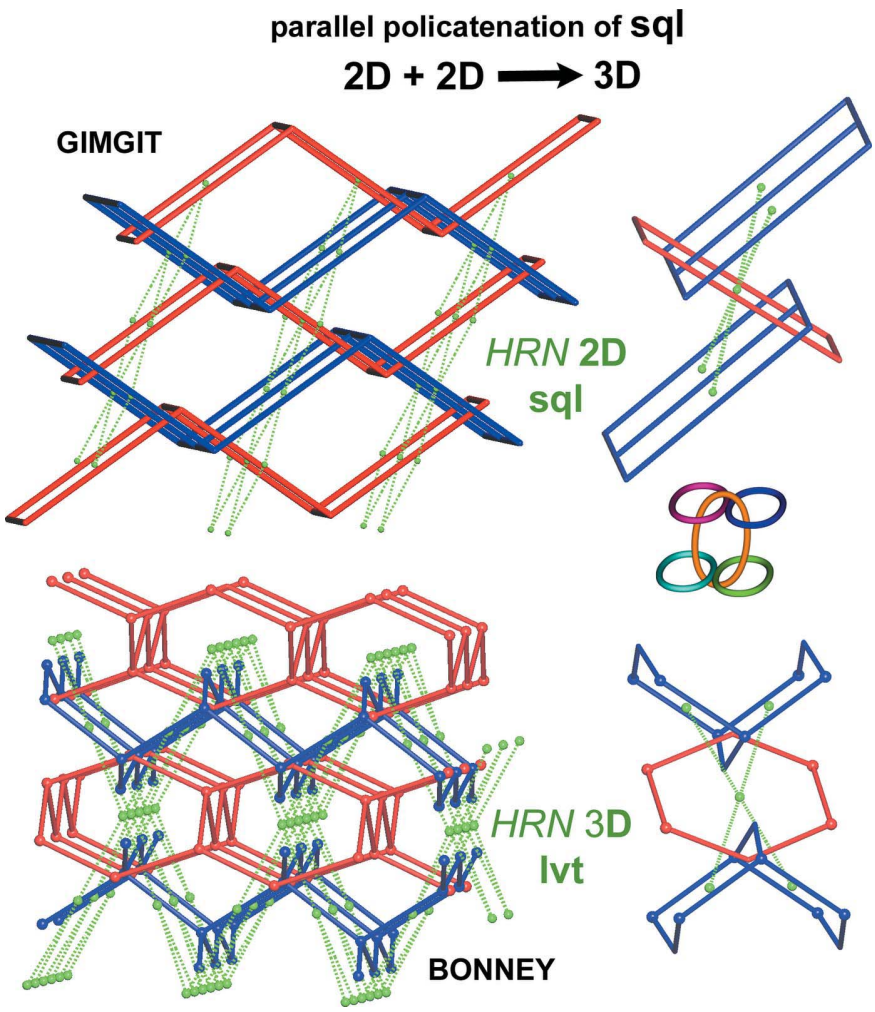

Figure 11

Parallel polycatenation in (top) GIMGIT and (bottom) BONNEY, BONNOI underlying nets and the corresponding HRNs, bouquets and HRN stars. 
(see Fig. 8), the polycatenation is realized by the presence of bent ligands represented by the additional 2-coordinated nodes on two opposite sides of the square. With these nodes the rings become 6-membered, but this extension does not influence the HRN topology.

If one distinguishes the HRN nodes corresponding to different entangling networks (for example, by assigning them different colours), the degree of catenation (Doc) and the index of separation (Is) can be easily determined. Indeed, Doc is equal to the number of other colours in the coordination shells of nodes of a particular colour, while $I s$ is equal to the number of groups of nodes of the same colour that should be removed to disjoint the HRN. In particular, BONNEY, BONNOI and GIMGIT are characterized by $D o c=2$ and $I s=$ 1 (Fig. 11). For inclined polycatenation only Doc is defined; it is 1 for ROZLIC and 2 for COFNOB and RONZOJ.

\subsection{Self-catenation}

Self-catenated nets are single nets that exhibit the peculiar feature of containing rings through which pass other components of the same network. In more detail, we must refer to the topological classification of nets, represented by their vertex symbols (Blatov et al., 2010); if one of the 'shortest rings' is catenated by other 'shortest rings' of the same net we can speak of a 'true' case of self-penetration. This is a necessary condition to be accomplished, since, otherwise, catenated rings or even knots can always be found in any kind of network, provided that sufficiently large circuits are considered. Unfortunately, such rigorous definition has not always been applied and nowadays we can find many papers describing not 'true' self-catenated networks (see e.g. Ke et al., 2011 and references therein). Description of the self-catenation phenomenon in terms of HRNs is similar to that of other types of catenation. As was mentioned above, we distinguish the edges of the HRNs corresponding to the links between rings of the same network and of different networks, so it is easy to separate the subnet describing self-catenation. The only problem is that not all self-catenated networks admit natural tilings; moreover, if the natural tiling exists, the catenated rings are always inessential and do not belong to the ring basis. This means that classification of catenation patterns can be difficult in some complicated cases: the ring basis should be

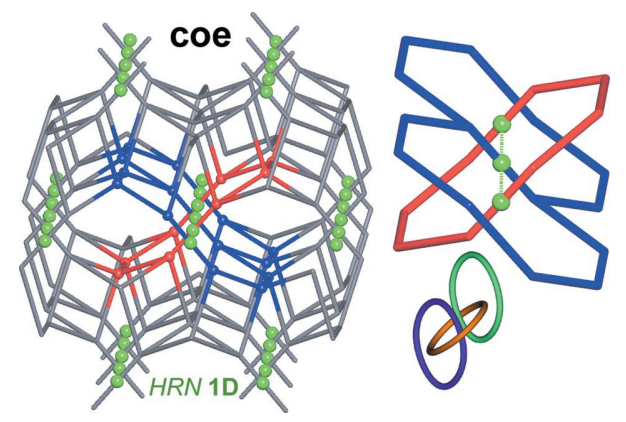

Figure 12

(Left) Self-catenated coesite (coe) network and the corresponding HRN; (right) bouquet and HRN star. chosen separately for the catenated rings. However, in most chemically reasonable cases, the ring basis can be easily chosen even manually. For example, in probably the most famous self-catenated network of coesite type [a silica polymorph, coe; see Carlucci et al. (2000) and references therein], the catenation occurs only between 8-rings, while the ring basis is formed by essential 4-, 6-, 9- and 10-rings (see the RCSR database). Nonetheless, the HRN is quite simple (chain-like, Fig. 12), and obviously the 8-rings are independent of each other (they form a basis of catenated rings).

Another example is the twt-type network that occurs in the chloroform solvate of $\left[\left(\mu_{2}-2,4,6\right.\right.$-tris(4-pyridyl)-1,3,5-triazine) $\mathrm{Ni}\left(\mathrm{NO}_{3}\right)_{2}$ ] (GOQWOY; Abrahams et al., 1999) and as twofold interpenetrating arrays in $\left[\left(\mu_{3}-4,4^{\prime}\right.\right.$-dicarboxy-2, $2^{\prime}$ bipyridine $\left.) M\left(\mathrm{H}_{2} \mathrm{O}\right)_{2}\right](M=\mathrm{Co}, \mathrm{Ni})$ (RAKBIO01, GAGWUH; Tynan et al., 2004), i.e. two types of entanglement, interpenetration and self-catenation, occur in the last two examples. In all the cases, the self-catenation is characterized by a 4-coordinated three-periodic HRN hxg-d-4-P6 $22-2$ (Fig. 13), which can be derived from 10-coordinated hxg-d by groupsubgroup relations (Blatov, 2007). The catenation pattern for the twofold arrays differs from those for self-dual networks and sphere packings; the corresponding HRN is $(14,20)$ coordinated. The ring basis for twt is well defined; it contains 12-rings of two kinds catenated by 14 and 20 12-rings of the
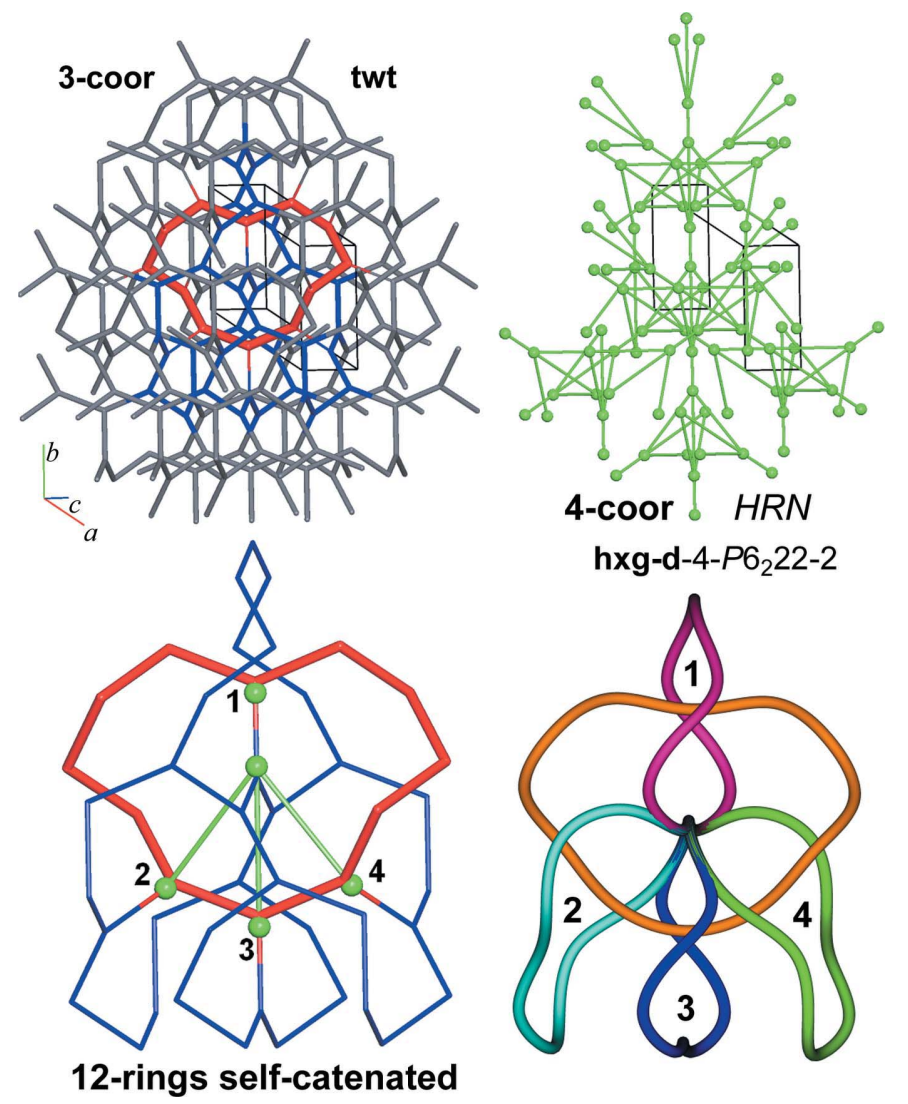

Figure 13

(Top) Self-catenated twt network and the corresponding HRN; (bottom) bouquets of catenating 12-rings and the corresponding HRN star. The catenated 12-ring is red, the four catenating 12-rings with numbers 1-4 are blue, and the HRN nodes (centres of 12-rings) are green. 
other network in the twofold array, while in the self-catenation the 12-rings of only one kind participate (Fig. 13). The selfcatenation patterns are the same in all three structures irrespective of additional interpenetration.

As can be seen from the two examples above, the study of HRNs in self-catenated nets is not straightforward, so a detailed analysis of self-catenation patterns in coordination networks will be the subject of future papers.

\section{Conclusion}

The Hopf ring nets (HRNs) are shown to be a rigorous method for identifying catenation patterns irrespective of the geometry of interweaving networks. We have demonstrated that HRNs are capable of representing different patterns within net arrays that are normally classified in the same interpenetration classes (Blatov et al., 2004) and also of analysing polycatenation [a much less studied area of the entanglement phenomenon (Proserpio, 2010)]. Our first application of the method will be to compute HRNs to classify the catenation patterns in 1000 reported interpenetrated coordination networks. For example, there are around 40 structures described as dia fourfold interpenetrated subdivided into five different classes that are described with only two different HRNs. Such taxonomy should help us gain a deeper insight into the nature of entanglement and to develop the design methods for new interlocking motifs.

This approach can easily be extended to other types of links between rings. In HRNs, such links are the simplest 2-ring (between two rings), while in the Borromean entanglement the links are 3-ring (Fig. 1), or, in general multi-ring (like the Brunnian interlocking). Such multi-ring links can be represented as additional multi-coordinated nodes in the resulting ring net. In this representation, a Hopf link corresponds to a trivial case of an additional 2-coordinated node of the ring net; such a node can be replaced by an edge without losing the information on connectivity (Fig. 1). Note, however, that such an extension is of mainly theoretical than practical importance since non-Hopf-type entanglements are quite rare in crystal structures. A further development of the method will be its extension to recognize not ambient-isotopic catenation patterns.

VAB is grateful for the 2009/2010 Fellowship from Cariplo Foundation and Landau Network - Centro Volta (Como, Italy). DMP thanks MIUR for financial support for the project PRIN 2008 'CRYSFORMS Design, properties and preparation of molecular crystals and co-crystals'.

\section{References}

Abrahams, B. F., Batten, S. R., Grannas, M. J., Hamit, H., Hoskins, B. F. \& Robson, R. (1999). Angew. Chem. Int. Ed. 38, 1475-1477. Alexandrov, E. V., Blatov, V. A., Kochetkov, A. V. \& Proserpio, D. M. (2011). CrystEngComm, 13, 3947-3958.

Allen, F. H. (2002). Acta Cryst. B58, 380-388.
Baburin, I. A. \& Blatov, V. A. (2007). Acta Cryst. B63, 791-802.

Baburin, I. A., Blatov, V. A., Carlucci, L., Ciani, G. \& Proserpio, D. M. (2005). J. Solid State Chem. 178, 2452-2474.

Baburin, I. A., Blatov, V. A., Carlucci, L., Ciani, G. \& Proserpio, D. M. (2008a). Cryst. Growth Des. 8, 519-539.

Baburin, I. A., Blatov, V. A., Carlucci, L., Ciani, G. \& Proserpio, D. M. (2008b). CrystEngComm, 10, 1822-1838.

Batten, S. R. (2001). CrystEngComm, 3, 67-72.

Batten, S. R. (2010). In Metal-Organic Frameworks: Design and Application, ch. 3, edited by L. R. MacGillivray. New York: Wiley.

Batten, S. R. \& Robson, R. (1998). Angew. Chem. Int. Ed. 37, 14601494.

Blatov, V. A. (2006). IUCr Compcomm Newsl. 7, 4-38.

Blatov, V. A. (2007). Acta Cryst. A63, 329-343.

Blatov, V. A., Carlucci, L., Ciani, G. \& Proserpio, D. M. (2004). CrystEngComm, 6, 377-395.

Blatov, V. A., Delgado-Friedrichs, O., O'Keeffe, M. \& Proserpio, D. M. (2007). Acta Cryst. A63, 418-425.

Blatov, V. A., O'Keeffe, M. \& Proserpio, D. M. (2010). CrystEngComm, 12, 44-48.

Blatov, V. A. \& Proserpio, D. M. (2009). Acta Cryst. A65, 202-212.

Carlucci, L., Ciani, G. \& Proserpio, D. M. (2003). Coord. Chem. Rev. 246, 247-289.

Carlucci, L., Ciani, G., Proserpio, D. M. \& Rizzato, S. (2000). J. Chem. Soc. Dalton Trans. pp. 3821-3827.

Delgado-Friedrichs, O., Foster, M. D., O'Keeffe, M., Proserpio, D. M., Treacy, M. M. J. \& Yaghi, O. M. (2005). J. Solid State Chem. 178, 2533-2554.

Delgado-Friedrichs, O. \& O'Keeffe, M. (2005). J. Solid State Chem. 178, 2480-2485.

Delgado-Friedrichs, O., O'Keeffe, M. \& Yaghi, O. M. (2003). Solid State Sci. 5, 73-78.

Delgado-Friedrichs, O., O'Keeffe, M. \& Yaghi, O. M. (2007). Phys. Chem. Chem. Phys. 9, 1035-1043.

Evans, O. R. \& Lin, W. (2001). Chem. Mater. 13, 3009-3017.

Fischer, W. \& Koch, E. (1976). Acta Cryst. A32, 225-232.

Goetzke, K. (1993). Bericht No. 9310. Christian-Albrechts University, Kiel, Germany.

Hoskins, B. F. \& Robson, R. (1990). J. Am. Chem. Soc. 112, 15461554.

Hyde, S. \& Delgado-Friedrichs, O. (2011). Solid State Sci. 13, 676-683.

Jensen, P., Price, D. J., Batten, S. R., Moubaraki, B. \& Murray, K. S. (2000). Chem. Eur. J. 6, 3186-3195.

Kang, Y., Zhang, J., Qin, Y.-Y., Li, Z.-J. \& Yao, Y.-G. (2007). J. Mol. Struct. 827, 126-129.

Ke, X.-J., Li, D.-D. \& Du, M. (2011). Inorg. Chem. Commun. 14, 788803.

Koch, E., Fischer, W. \& Sowa, H. (2006). Acta Cryst. A62, 152-167.

Mahenthirarajah, T., Li, Y. \& Lightfoot, P. (2009). Dalton Trans. pp. 3280-3285.

Martin, D. P., Supkowski, R. M. \& LaDuca, R. L. (2008). Polyhedron, 27, 2545-2550.

O'Keeffe, M., Peskov, M. A., Ramsden, S. J. \& Yaghi, O. M. (2008). Acc. Chem. Res. 41, 1782-1789.

Proserpio, D. M. (2010). Nat. Chem. 2, 435-436.

Soma, T. \& Iwamoto, T. (1996). J. Inclusion Phenom. Mol. Recognit. Chem. 26, 161-173.

Tian, Z., Lin, J., Su, Y., Wen, L., Liu, Y., Zhu, H. \& Meng, Q.-J. (2007). Cryst. Growth Des. 7, 1863-1867.

Tynan, E., Jensen, P., Kelly, N. R., Kruger, P. E., Lees, A. C., Moubaraki, B. \& Murray, K. S. (2004). Dalton Trans. pp. 3440-3447.

Wang, G.-H., Li, Z.-G., Jia, H.-Q., Hu, N.-H. \& Xu, J.-W. (2009). CrystEngComm, 11, 292-297.

Wells, A. F. (1954). Acta Cryst. 7, 849-853.

Xiao, H.-P., Wang, J.-G., Li, X.-H. \& Morsali, A. (2005). Z. Anorg. Allg. Chem. 631, 2976-2978. 